\title{
Tunable topological states hosted by unconventional superconductors with adatoms
}

\author{
Andreas Kreisel $\odot,{ }^{1}$ Timo Hyart, ${ }^{2,3}$ and Bernd Rosenow ${ }^{1}$ \\ ${ }^{1}$ Institut für Theoretische Physik, Universität Leipzig, Brüderstrasse 16, 04103 Leipzig, Germany \\ ${ }^{2}$ International Research Centre MagTop, Institute of Physics, \\ Polish Academy of Sciences, Aleja Lotnikow 32/46, PL-02668 Warsaw, Poland \\ ${ }^{3}$ Department of Applied Physics, Aalto University, 00076 Aalto, Espoo, Finland
}

(Received 15 April 2021; revised 3 June 2021; accepted 3 June 2021; published 14 July 2021)

\begin{abstract}
Chains of magnetic atoms, placed on the surface of $s$-wave superconductors, have been established as a laboratory for the study of Majorana bound states. In such systems, the breaking of time reversal due to magnetic moments gives rise to the formation of in-gap states, which hybridize to form one-dimensional topological superconductors. However, in unconventional superconductors even nonmagnetic impurities induce in-gap states since scattering of Cooper pairs change their momentum but not their phase. Here we propose a path for creating topological superconductivity, which is based on an unconventional superconductor with a chain of nonmagnetic adatoms on its surface. The topological phase can be reached by tuning the magnitude and direction of a Zeeman field, such that Majorana zero modes at its boundary can be generated, moved, and fused. To demonstrate the feasibility of this platform, we develop a general mapping of films with adatom chains to one-dimensional lattice Hamiltonians. This allows us to study unconventional superconductors such as $\mathrm{Sr}_{2} \mathrm{RuO}_{4}$ exhibiting multiple bands and an anisotropic order parameter.
\end{abstract}

DOI: 10.1103/PhysRevResearch.3.033049

\section{INTRODUCTION}

Combining topology and superconductivity has been heralded as a new paradigm for the realization of exotic new particles-Majorana zero modes (MZMs) --whose nonAbelian braiding statistics would enable fault-tolerant quantum computations [1,2]. Moreover, the existence of MZMs is topologically protected, making them inert to disorder effects. To-date, two main approaches, based on the Kitaev chain [3], have been pursued in the quest for topological superconductors. In the first approach $s$-wave superconductivity is proximity induced in nanowires with strong spin-orbit coupling [4-7], while in the second approach the hybridization of impurity (Shiba) bound states gives rise to a topologically nontrivial superconducting phase [8-13]. Experimentally, the latter has been realized by placing a chain of magnetic atoms on the surface of an $s$-wave superconductor [14-18]. In these platforms, evidences for MZMs at the endpoints of the system were found in transport measurements on nanowires [6,7] and in scanning tunneling spectroscopy on Shiba chains [15-19]. While a number of possibilities of chains of addatoms of potential scatterers, magnetic scatterers, and nanowires on top of superconductors have been investigated theoretically [20-24] and proposals for moving, fusing, and braiding the MZMs have been brought forward [25-28], it remains an open challenge experimentally to implement those.

Published by the American Physical Society under the terms of the Creative Commons Attribution 4.0 International license. Further distribution of this work must maintain attribution to the author(s) and the published article's title, journal citation, and DOI.
In this work we propose a path to realize one-dimensional topological superconductivity by placing nonmagnetic atoms on the surface of an unconventional triplet superconductor, see Fig. 1. The key advantage of our proposal is that it is possible to move and fuse MZMs by controlling a magnetic Zeeman field. Since candidate systems for the realization of triplet superconductivity usually exhibit multiple bands, we go beyond a single-band description and use a model for $\mathrm{Sr}_{2} \mathrm{RuO}_{4}$ to demonstrate that our method can easily be applied to multiband superconductivity. At the same time, we note that $\mathrm{Sr}_{2} \mathrm{RuO}_{4}$ has been subject to intense theoretical and experimental investigations regarding the nature of the superconducting pairing [29-33]. Implementing our proposal in candidate systems for triplet superconductivity such as $\mathrm{UPt}_{3}[34,35], \mathrm{UTe}_{2}$ [36,37], and $\mathrm{LaNiGa}_{2}$ [38] could establish a new MZM platform and improve the understanding of the pairing symmetries in these systems.

\section{MODEL}

\section{A. Bulk superconductor}

Our starting point is a single-band Hamiltonian

$$
H=H_{\mathrm{BdG}}+H_{\mathrm{Z}},
$$

where $H_{\mathrm{BdG}}$ describes a bulk triplet superconductor on a twodimensional lattice, and $H_{\mathrm{Z}}$ is the Zeeman term in an external field. In momentum space it has the matrix structure

$$
H(\mathbf{p})=\left(\begin{array}{cc}
h(\mathbf{p}) & \Delta(\mathbf{p}) \\
\Delta(\mathbf{p})^{\dagger} & -h(-\mathbf{p})^{T}
\end{array}\right),
$$

where $h(\mathbf{p})=\xi(\mathbf{p}) \cdot \sigma_{0}+\mathbf{h} \cdot \boldsymbol{\sigma}, \quad \xi(\mathbf{p}) \quad$ is the energymomentum dispersion, $\mathbf{h}$ is the Zeeman field, and $\sigma_{0}$ and $\sigma$ are the unit matrix and Pauli matrices acting in spin space. 
In the following we measure all energies in units of the Fermi energy $E_{F}$. In the off-diagonal, $\Delta(\mathbf{p})=i[\mathbf{d}(\mathbf{p}) \cdot \boldsymbol{\sigma}] \sigma_{y}$ is the pairing term, whose minimum absolute value on the Fermi surface we denote by $\Delta_{\text {min }}$; for details see Appendix A. Here $\mathbf{d}(\mathbf{p})$ describes the vector order parameter of the triplet superconductor. Here we concentrate on the case of a helical $p$-wave order parameter

$$
\mathbf{d}_{h}=-i \Delta_{t}\left(\mathbf{e}_{x} \sin p_{y}+\mathbf{e}_{y} \sin p_{x}\right),
$$

and in the Supplemental Material [40] we discuss the generalizations to a chiral $p$-wave order parameter and multiband models using $\mathrm{Sr}_{2} \mathrm{RuO}_{4}$ as an example.

\section{B. Chain of nonmagnetic impurities}

The chain of atoms placed along the $x$ direction $\mathbf{r}_{n}=n a_{0} \mathbf{e}_{x}$ (with integer $n$ ) is described by

$$
H_{\mathrm{imp}}=\hat{U} \sum_{n} \delta_{\mathbf{r}, \mathbf{r}_{n}},
$$

with the matrix $\hat{U}=V_{\mathrm{imp}} \tau_{z} \sigma_{0}$ mediating nonmagnetic impu-

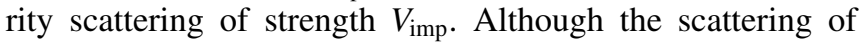
Bogoliubov quasiparticles preserves spin, there are still Shiba in-gap states in this system because the scattering does not change the phase in order to match the $p$-wave momentum dependence of the order parameter. In the case of a chain, Shiba states localized in the vicinity of the impurity atoms hybridize and give rise to impurity bands within the bulk gap $\Delta_{\text {min }}$ of the superconductor. These bands can be accurately described by an effective Hamiltonian

$$
H_{\text {eff }}\left(k_{x}\right)=\hat{U}^{-1} \tilde{G}^{-1}\left[G_{I}\left(k_{x}\right) \hat{U}-1\right],
$$

which depends on the momentum $k_{x}$ in a supercell Brillouin zone with lattice constant $a_{0}$. We derive the matrices on the right-hand side by linearizing the bulk Green function with respect to energy, and obtain $G_{I}\left(k_{x}\right)$, which describes the propagation of Bogoliubov quasiparticles between the impurities, by Fourier transforming the bulk Green function at the impurity sites. The matrix $\tilde{G}^{-1}$ enters as a prefactor and contains the renormalization of the bandwidth (see Appendix B).

\section{EFFECTIVE HAMILTONIAN}

The mapping onto the effective Hamiltonian Eq. (5) can be understood as integrating out the quantum numbers $p_{y}$ of momenta perpendicular to the chain. Therefore, in the absence of a Zeeman field the effective Hamiltonian has the structure

$$
H_{\mathrm{eff}}^{0}\left(k_{x}\right)=\xi_{\mathrm{eff}}\left(k_{x}\right) \tau_{z} \sigma_{0}+\Delta_{\mathrm{eff}}\left(k_{x}\right) \tau_{x} \sigma_{0},
$$

diagonal in spin space, and describing twofold degenerate impurity bands inside the bulk superconducting gap. Here $\xi_{\text {eff }}\left(k_{x}\right)$ and $\Delta_{\text {eff }}\left(k_{x}\right)$ are the effective dispersion and pairing for the impurity chain, containing further-neighbor coupling terms between the impurity states.

The Hamiltonian $H_{\text {eff }}^{0}\left(k_{x}\right)$ is time-reversal symmetric, so that the system can support a topological phase with an even number of MZMs at each end of the chain [39]. We can realize unpaired MZMs by additionally breaking the symmetry down to only particle-hole symmetry by use of a Zeeman field

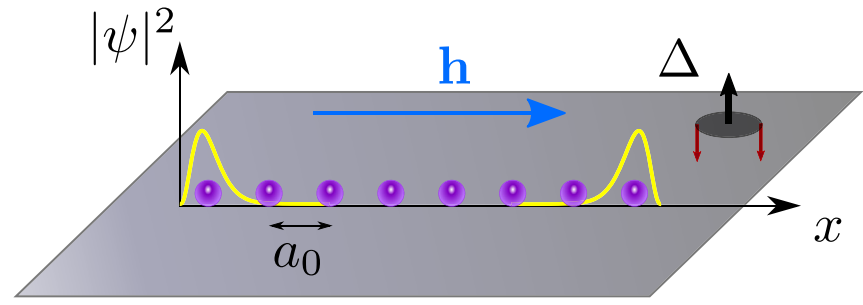

FIG. 1. Setup: Nonmagnetic adatoms (purple balls) are placed at a distance $a_{0}$ to form a chain on the surface of a helical triplet superconductor with order parameter $\Delta$. The Cooper pairs exhibit equal spin (red arrows), and their orbital angular momentum (black arrow) points opposite to the spin direction. An external Zeeman field $\mathbf{h}$ can be used to tune the system into the topological phase supporting Majorana zero modes at the endpoints of the chain as sketched by the yellow plot of the magnitude $|\psi|^{2}$ of the wave function.

such that the system, when tuned into the topological phase, exhibits unpaired MZMs at the end of the impurity chain A Zeeman field in $z$ direction can be described by $H_{\mathrm{eff}, z}^{\mathrm{Z}}=$ $h_{\mathrm{eff}, z} \sigma_{z} \tau_{z}$ with a renormalized magnitude $h_{\mathrm{eff}, z}$, which can be calculated by including chemical potential shifts $\xi(\mathbf{p}) \pm h_{z}$ in the bulk dispersion of the spin up and down electrons, respectively. We find that $h_{\text {eff, } z} \ll h_{z}$ because the energy of Shiba in-gap state depends only weakly on the chemical potential (see the Supplemental Material [40]). Thus, the effective Hamiltonian is diagonal in spin space with each block $\tilde{H}_{\text {eff } \pm}=$ $\left[\xi_{\text {eff }}\left(k_{x}\right) \pm h_{\text {eff }, z}\right] \tau_{z}+\Delta_{\text {eff }}\left(k_{x}\right) \tau_{x}$ exhibiting the same topological properties as the Kitaev chain. The effective field $\pm h_{\mathrm{eff}, z}$ plays the role of the chemical potential, which can drive a topological phase transition. However, since the effective field $h_{\mathrm{eff}, z}$ is parametrically small, the topological phase can only be reached by fine tuning the impurity strength $V_{\mathrm{imp}}$ such that the impurity bands almost touch zero already without a Zeeman field.

For the Zeeman field pointing in $y$ direction, the additional term to the effective Hamiltonian is $H_{\mathrm{eff}, y}^{\mathrm{Z}}=h_{\mathrm{eff}, y} \sigma_{y} \tau_{0}$. In this case, the two BdG bands are shifted trivially in energy with respect to each other, leaving their topological character unchanged, i.e., a field in $y$ direction cannot tune into the topological phase.

Finally, for a field in $x$ direction, the Zeeman term reads $H_{\mathrm{eff}, x}^{\mathrm{Z}}=h_{\mathrm{eff}, x} \tau_{z} \sigma_{x}$ with weak renormalization of the effective Zeeman field from the bulk value $h_{\text {eff, } x} \sim h_{x}$. The effective Hamiltonian can now be rotated around the $y$ axis in spin space $\sigma_{x} \rightarrow \sigma_{z}$, such that in the new basis the effective field $h_{\text {eff }, x}$ plays again the role of a chemical potential. The difference to the case of a Zeeman field in $z$ direction is that the weakly renormalized $h_{\mathrm{eff}, x}$ can drive a topological phase transition much more efficiently than the strongly reduced $h_{\text {eff }, z}$ discussed above.

\section{TOPOLOGICAL PHASE DIAGRAM}

To quantitatively demonstrate the tunability of topological superconductivity, we compute the topological phase diagram (see Figs. 2 and 3) as characterized by the topological 
(a) single band

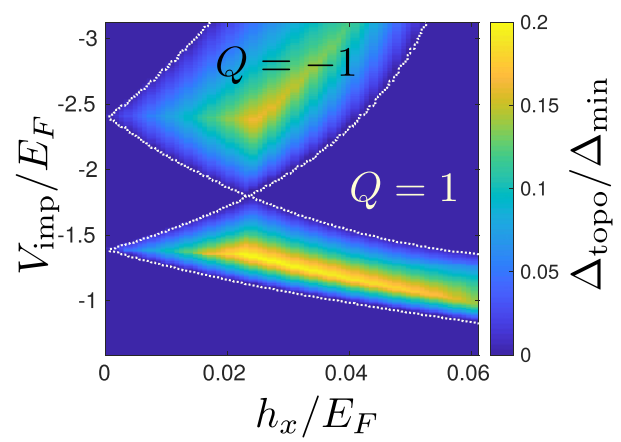

(b) $\mathrm{Sr}_{2} \mathrm{RuO}_{4}$

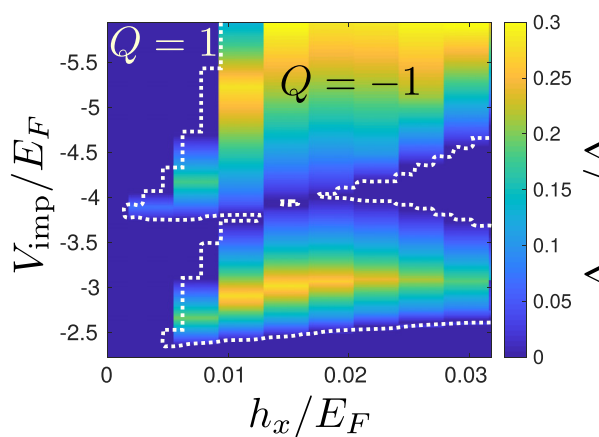

(c)

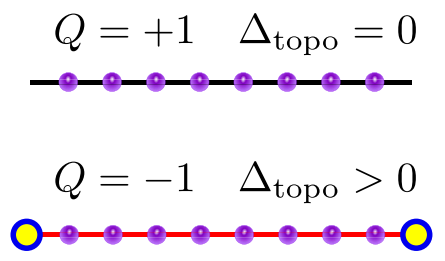

FIG. 2. Topological phase diagram. (a) Helical single-band superconductor: the topological phase $(Q=-1$ with finite topological gap $\Delta_{\text {topo }}$ ) can be reached for a wide range of impurity strengths $V_{\text {imp }}$ by tuning an external Zeeman field $h_{x}$. The phase boundary is marked with a white dashed line. An energy gap $\Delta_{\text {topo }} \approx \Delta_{\min } / 5$ can be reached in the topological phase, leading to well-localized MZMs at the length scale $\zeta / a_{0} \approx \Delta_{\text {min }} / \Delta_{\text {topo }}$. Parameters are $\Delta_{0} / E_{F} \approx 0.08, a_{0} \approx 2.5 \xi$. (b) Multiband model for $\mathrm{Sr}_{2} \mathrm{RuO}_{4}$ with a realistic superconducting order parameter (see the Supplemental Material [40]) shows similar tunability as the single-band model, with a maximum gap $\Delta_{\text {topo }} \approx \Delta_{\text {min }} / 3$. (c) For the topological invariant $Q=+1$ the system is in the trivial phase (and we denote $\Delta_{\text {topo }}=0$ ), while for $Q=-1$ MZMs at the ends of the impurity chain exist and are protected against perturbations of the order of the topological gap $\Delta_{\text {topo }}$.

invariant

$$
Q=\prod_{k_{x} \in \text { TRIM }} Q\left(k_{x}\right), \quad Q\left(k_{x}\right)=\operatorname{sgn}\left(\operatorname{Pf}\left[H\left(k_{x}\right) \tau_{x}\right]\right) .
$$

It is given as the product of Pfaffians $Q\left(k_{x}\right)$ at the time-reversal invariant momenta of the corresponding onedimensional Brillouin zone, details on its derivation can be found in Appendix C. We supplement the fully numerical supercell calculation by computing the topological invariant also using $Q_{\text {eff }}\left(k_{x}\right)=\operatorname{sgn}\left(\operatorname{Pf}\left[H_{\text {eff }}\left(k_{x}\right) \tau_{x}\right]\right)$. The excellent agreement between $Q_{\text {eff }}$ and $Q$ (see the Supplemental Material [40]) indicates that $H_{\text {eff }}\left(k_{x}\right)$ indeed faithfully describes the low-energy physics of the impurity chain. In the nontrivial case $Q=-1$ we define the topological gap $\Delta_{\text {topo }}$ as the minimum of the eigenenergies of $H\left(k_{x}\right)$ in the Brillouin zone, see Appendix D. To detect the non-Abelian properties of the MZMs, it is necessary that the coupling between MZMs is weak. Thus, the distance between neighboring MZMs needs to be much larger than $\zeta \approx \hbar v_{F \text {,eff }} / \Delta_{\text {topo }}$, where $v_{F \text {,eff }}$ is the Fermi velocity for the impurity band. An estimate of $v_{F \text {,eff }}$ yields $\zeta / a_{0} \approx \Delta_{\min } / \Delta_{\text {topo }}$. In order to avoid thermal excitations, the temperature needs to be smaller than the topological gap $k_{\mathrm{B}} T<\Delta_{\text {topo }}$.

In Fig. 2 we present the topological phase diagram for the single-band model and for a multiband description of $\mathrm{Sr}_{2} \mathrm{RuO}_{4}$, revealing that the topological phase can be reached in both cases by application of a Zeeman field $h_{x}$ in the direction along the impurity chain for a large range of the impurity potential $V_{\text {imp }}$. Experimentally, suitable adatoms can be identified by examining the appearance of in-gap states in the tunneling spectra. We summarize that our proposal might be feasible in a helical $p$-wave superconductor where impurity adatoms can be controlled experimentally. The multiband helical $p$-wave order parameter considered in Fig. 2 is one of the possible candidate pairing symmetries for $\mathrm{Sr}_{2} \mathrm{RuO}_{4}$ [32] Controllable placing of adatoms might be facilitated by step (a)

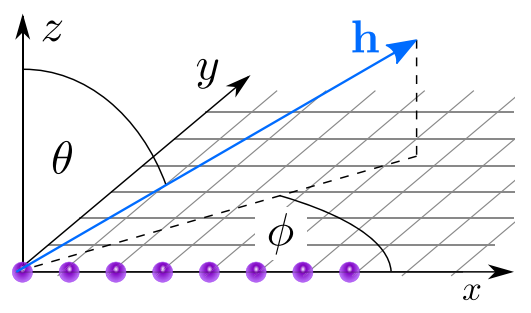

(b) single band

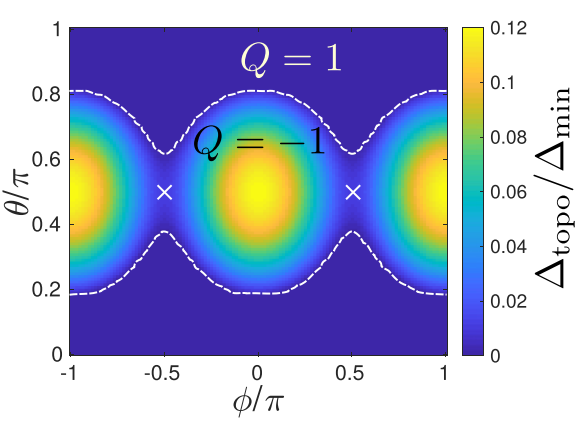

(c) $\mathrm{Sr}_{2} \mathrm{RuO}_{4}$

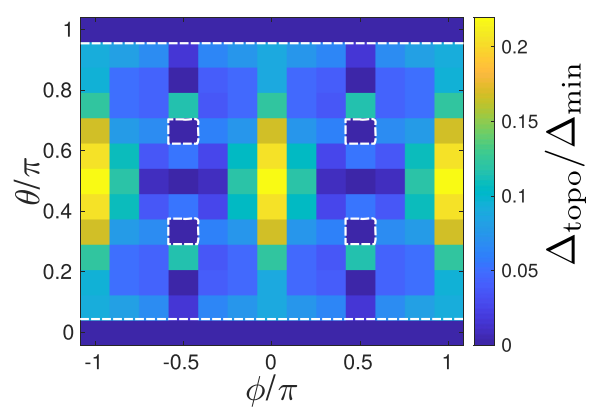

FIG. 3. Field direction dependence of the topological gap. (a) The direction of the Zeeman field relative to the direction of the impurity chain is parametrized by the azimuthal angle $\phi$ and the polar angle $\theta$. Changing the direction of the field allows us to enter or leave the topological phase. (b) Topological phase diagram for a single-band system with parameters $V_{\text {imp }} / E_{F}=-1.2,|h| / E_{F}=0.03, a_{0} \approx 2.5 \xi$; the white dashed line is the boundary between topological and trivial phase. (c) Similar phase diagram for $\operatorname{Sr}_{2} \mathrm{RuO}_{4}\left(V_{\mathrm{imp}} / E_{F}=-5.2,|h| / E_{F}=\right.$ $\left.0.015, a_{0} \approx 0.8 \xi\right)$. In both cases, the topological gap $\Delta_{\text {topo }}$ is maximal for the field along the impurity chain $(\phi=0, \theta=\pi / 2)$, and the topologically trivial phase can be reached by either tuning to $\phi=\pi / 2$, or towards $\theta=0, \pi$, i.e., to a transverse directions relative to the chain. The isolated point at which the system remains gapless (white cross) corresponds to an in-plane field perpendicular to the chain. 


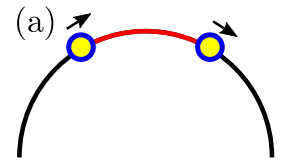

rotate $\vec{h}$ (b)

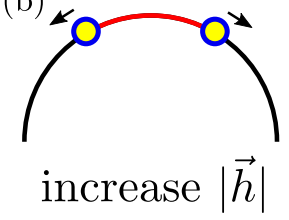

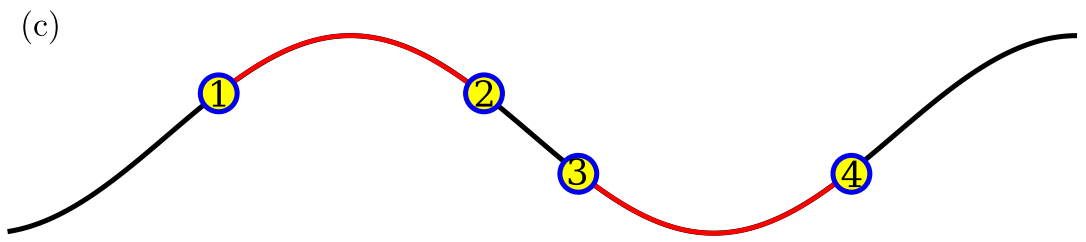

FIG. 4. Moving and fusion of Majorana zero modes. A curved impurity chain might be tuned partially into the topological state (red arcs) if the the angle between the Zeeman field and the local direction of the chain puts it into the topological phase. MZMs (yellow dots) occur at the boundaries of trivial and nontrivial regions, which can be moved in two different ways: (a) Rotating the field around the axis perpendicular to the plane moves the Majoranas in the same direction, while (b) changing the field magnitude or polar angle can move them in opposite directions. (c) In a wiggly impurity chain MZMs 1 and 2, 3 and 4 can be created pairwise from the vacuum by changing the field magnitude, and by tuning deeper into the topological phase one eventually fuses the MZMs 2 and 3.

edges, and in the Supplemental Material [40] we show that the topological phase can be reached by application of a Zeeman field also in this case. In Fig. 3 we illustrate that the direction of the Zeeman field can be used to tune a system into and out of the topological phase. Namely, tuning the azimuthal angle $\phi$ has a strong effect on the topological gap $\Delta_{\text {topo }}$. A similar analysis of the tunability of a system with chiral $p$-wave order parameter (see the Supplemental Material [40]) reveals that rotating the Zeeman field within the $x-y$ plane has no effect at all. Hence, this difference in behavior could be used to experimentally diagnose and discriminate the helical $p$ wave from a chiral $p$-wave order parameter, an important question for example in the $\mathrm{Sr}_{2} \mathrm{RuO}_{4}$ system [29-32].

\section{DISCUSSION}

For the case of magnetic adatoms, the dependence of adatom magnetic order on an external Zeeman field has been suggested as a means to to create, braid, and fuse MZMs [27]. Here we exploit the direct dependence of the topological phase diagram on the Zeeman field (see Fig. 3). So far we have arbitrarily chosen that the impurity chain is oriented along the $x$ axis, and as a result a Zeeman field in $x$ direction was most suitable to induce a topological phase. More generally however, the relevant parameter is the relative angle of the Zeeman field with the impurity chain, and for a curved chain the relevant angle would be the angle between the field and the local tangential direction as defined in Fig. 3(a). Thus, MZMs on curved impurity chains are located at all interfaces between trivial and nontrivial regions, i.e., at positions where the local tangent and the external field draw a critical angle, see Fig. 4. Hence, MZMs can be moved along the impurity chain by either rotating the direction of the Zeeman field or by changing the magnitude of the field, which modifies the critical angle.

Increasing the Zeeman field along a wiggly impurity chain creates two pairs of Majoranas which can formally be described by operators $\gamma_{i}, i=1,2,3,4$, satisfying $\gamma_{i}=\gamma_{i}^{\dagger}$ and anticommutation relations $\left\{\gamma_{i}, \gamma_{j}\right\}=2 \delta_{i j}$ [for the labeling of MZMs see Fig. 4(c)]. Grouping the MZMs in pairs of two, we can define the left and right number operators $n_{l}=\frac{1}{2}(1+$ $\left.i \gamma_{1} \gamma_{2}\right)$ and $n_{r}=\frac{1}{2}\left(1+i \gamma_{3} \gamma_{4}\right)$ with eigenvalues 0 and 1 , and define a Hilbert space spanned by basis states $\left|n_{l} n_{r}\right\rangle$. Creating the MZMs from the vacuum, the initial state is given by $|\psi\rangle=|00\rangle$ in this basis. Tuning deeper into the topological phase, the inner MZMs 2 and 3 will fuse such that the final state will be a statistical mixture of 0 and 1 for the operator $n_{o}=\frac{1}{2}\left(1+i \gamma_{1} \gamma_{4}\right)$ (see the Supplemental Material [40]). In the fusion process the projective measurement can be performed by detecting the charge acquired by MZMs 2 and 3 after they have hybridized [28]. The movement and projective measurements are the key ingredients for manipulation of MZMs, and they can be realized by controlling external magnetic field as discussed above. However, we also need to preserve the quantum information stored in the MZMs. In the Supplementary Material [40] we discuss the corresponding requirements and propose to manipulate the local magnetization by spintronic means to obtain signatures of non-Abelian statistics of MZMs.

In summary, we have shown that topological superconductivity can be realized by placing nonmagnetic adatoms on the surface of an unconventional superconductor, and that the topological invariant can be controlled with the magnitude and direction of a Zeeman field. Our considerations are based on a lattice Hamiltonian which can describe materials exhibiting a complex structure of the order parameter and multiple bands. We have identified the field direction which can most efficiently tune the system into the topological phase and we have proposed a scheme to move and fuse MZMs. An experimental realization of this proposal could become a scalable platform for topological quantum information processing based on the non-Abelian statistics of MZMs.

\section{ACKNOWLEDGMENTS}

The research was partially supported by the Foundation for Polish Science through the IRA Programme co-financed by EU within SG OP. We acknowledge support from Leipzig University for Open Access Publishing. The code to numerically calculate the spectra and topological invariants discussed in this paper as well as the data that has been used to generate the plots within this paper is available from the corresponding author upon reasonable request. B.R. acknowledges support by DFG Grant No. RO 2247/11-1.

\section{APPENDIX A: TIGHT-BINDING MODEL}

For the single-band model, we use the normal state dispersion $\xi(\mathbf{p})=-2 t\left(\cos p_{x}+\cos p_{y}\right)-\mu$ on a square lattice, where $t$ is the nearest-neighbor hopping and $\mu \approx-1.44 t$ is the chemical potential fixed such that the filling is one quarter and the Fermi energy $E_{F} \approx 2.56 t$. 
The superconducting order parameter can be written in real space as

$$
\Delta=\sum_{i j} \sum_{\alpha \beta} \sum_{\sigma \sigma^{\prime}} \Delta_{i j, \alpha \beta, \sigma \sigma^{\prime}} c_{i, \alpha, \sigma}^{\dagger} c_{j, \beta, \sigma^{\prime}}^{\dagger}+\text { H.c. }
$$

For the single-band system with a triplet order parameter, the coefficients read $\Delta_{i j, \alpha \beta, \sigma \sigma^{\prime}}=\Delta_{0} \mathbf{d}_{i, j} \cdot \boldsymbol{\sigma} i \sigma_{y}$ with the Pauli operators $\boldsymbol{\sigma}=\left[\sigma_{x}, \sigma_{y}, \sigma_{z}\right]$ and a vector $\mathbf{d}_{i, j}$. In the main text we consider the physical consequences of the helical $p$-wave order parameter $\mathbf{d}=-i \Delta_{t}\left(\sin p_{y} \mathbf{e}_{x}+\sin p_{x} \mathbf{e}_{y}\right)$ which in real space leads to nearest-neighbor pairings $\Delta_{0}=\delta_{i}\left(\begin{array}{cc}-1 & 0 \\ 0 & 1\end{array}\right)$ with $\delta_{i}=\mp \Delta_{t} / 2$ for the relative lattice vector $(0, \pm 1)$, and $\Delta_{0}=$ $\delta_{i}\left(\begin{array}{cc}i & 0 \\ 0 & i\end{array}\right)$ with $\delta_{i}=\mp \Delta_{t} / 2$ for the relative lattice vector $( \pm 1,0)$.

The multiband model for $\mathrm{Sr}_{2} \mathrm{RuO}_{4}$ [41] is discussed in the Supplemental Material [40].

\section{APPENDIX B: GREEN FUNCTION APPROACH AND EFFECTIVE HAMILTONIAN}

Derivations of effective Hamiltonians for continuum models have been worked out in detail for example for helical Shiba chains in Ref. [10] and spinless superconductors [22]. Here we generalize this approach to multiband lattice models and derive the effective Hamiltonian for the impurity bands as cited in Eq. (5) of the main text. Starting point is the eigenvalue equation of the Bogoliubov-de Gennes Hamiltonian (including the impurity chain) $\left(H_{\mathrm{BdG}}+H_{\mathrm{Z}}+H_{\text {imp }}\right) \Psi=E \Psi$ with the eigenstate $\Psi$ and eigenenergy $E$. Next, we introduce the Green function operator of the bulk system

$$
G(E)=\left(E-H_{\mathrm{BdG}}-H_{\mathrm{Z}}\right)^{-1}
$$

to obtain a nonlinear eigenproblem

$$
\Psi=G(E) H_{\mathrm{imp}} \Psi .
$$

Evaluating Eq. (B2) only at the impurity sites $\mathbf{r}_{m}=a_{0} m \mathbf{e}_{x}$, and using that the impurity Hamiltonian [Eq. (4)] is diagonal, we obtain

$$
\Psi\left(\mathbf{r}_{m}\right)=\sum_{\mathbf{r}_{n}} G\left(E, \mathbf{r}_{m}-\mathbf{r}_{n}\right) \hat{U} \Psi\left(\mathbf{r}_{n}\right) .
$$

Because of the periodicity with respect to translations by the impurity chain lattice vector $a_{0} \mathbf{e}_{x}$, it is useful to transform this equation to momentum space (with respect to the supercell) $\Psi\left(k_{x}\right)=\sum_{m} \Psi\left(\mathbf{r}_{m}\right) e^{-i k_{x} a_{0} m}$ such that the eigenvalue equation can be rewritten as

$$
\Psi\left(k_{x}\right)=\sum_{n} G\left(E, \mathbf{r}_{n}\right) e^{-i k_{x} a_{0} n} \hat{U} \Psi\left(k_{x}\right) .
$$

The real space Green function can be obtained via its Fourier representation

$$
\begin{aligned}
& G(E, \mathbf{r})=\frac{1}{\Omega_{\mathrm{BZ}}} \int_{\mathrm{BZ}} d^{2} p G(E, \mathbf{p}) e^{i \mathbf{p} \cdot \mathbf{r}}, \\
& G(E, \mathbf{p})=\left[E-H_{\mathrm{BdG}}(\mathbf{p})-H_{Z}\right]^{-1},
\end{aligned}
$$

where the integral is over the bulk Brillouin zone with momentum space area $\Omega_{\mathrm{BZ}}$. By linearizing the bulk Green function at $E=0$, we obtain

$$
G(E, \mathbf{p})=G(0, \mathbf{p})-E \tilde{G}(\mathbf{p}),
$$

where $\quad G(0, \mathbf{p})=-\left[H_{\mathrm{BdG}}(\mathbf{p})+H_{\mathrm{Z}}\right]^{-1}$ and $\quad \tilde{G}(\mathbf{p})=$ $\left[H_{\mathrm{BdG}}(\mathbf{p})+H_{\mathrm{Z}}\right]^{-2}$ exist for fully gapped systems. Furthermore, we keep the linear correction $\propto E$ only in the on-site term of the Green function to obtain

$$
G\left(E, \mathbf{r}_{n}\right)=G\left(0, \mathbf{r}_{n}\right)-E \delta_{\mathbf{r}_{n}, 0} \tilde{G},
$$

where

$$
\tilde{G}=\frac{1}{\Omega_{\mathrm{BZ}}} \int_{\mathrm{BZ}} d^{2} p\left[H_{\mathrm{BdG}}(\mathbf{p})+H_{\mathrm{Z}}\right]^{-2} .
$$

We now insert Eqs. (B4), (B5), and (B6) into Eq. (B3) and introduce the Fourier transforms with respect to the supercell as

$$
G_{I}\left(k_{x}\right)=\sum_{n} G\left(0, \mathbf{r}_{n}\right) e^{-i a_{0} n k_{x}}
$$

to obtain

$$
\Psi\left(k_{x}\right)=\left[G_{I}\left(k_{x}\right) \hat{U}-E \tilde{G} \hat{U}\right] \Psi\left(k_{x}\right) .
$$

Rearranging the terms and multiplication with inverse matrices brings the eigenvalue equation in the form

$$
H_{\text {eff }}\left(k_{x}\right) \Psi\left(k_{x}\right)=E \Psi\left(k_{x}\right),
$$

where the effective Hamiltonian is

$$
H_{\text {eff }}\left(k_{x}\right)=\hat{U}^{-1} \tilde{G}^{-1}\left[G_{I}\left(k_{x}\right) \hat{U}-1\right] .
$$

This Hamiltonian becomes exact at $E=0$ where the topological phase transition occurs and therefore can be used to determine the phase diagram exactly.

This approach is general and can be applied to all lattice Hamiltonians. In this work we have applied it to the single-band $p$-wave superconductors and multiband model for $\mathrm{Sr}_{2} \mathrm{RuO}_{4}$ [41], but similar theoretical investigations can be performed also for other candidate materials for multiband triplet superconductors [34-38].

\section{APPENDIX C: TOPOLOGICAL INVARIANT}

A finite magnetic field breaks time-reversal symmetry such that the remaining symmetry of the Hamiltonian, Eq. (1), is particle-hole symmetry, described by a particle-hole operator $P$ anticommuting with the Hamiltonian $\{H, P\}=0$. The superconducting pairing has the property $\Delta^{T}=-\Delta$ and the normal state block is Hermitian, $h^{\dagger}=h$. Therefore, $P=\tau_{x} K$ is the desired anticommuting operator, where $\tau_{x}$ is the Pauli matrix in particle-hole space and $K$ is the complex conjugation. The parity operator $\hat{P}=(-1)^{\hat{N}}$, with $\hat{N}$ being the particle number operator, commutes with the Hamiltonian $[H, \hat{P}]=0$, thus there is a common system of eigenstates. Since the parity operator has the eigenvalues \pm 1 , the ground state of the system is either of odd or even parity. The parity can be calculated by Eq. (7), where we formally have factorized out a prefactor of $(-1)^{n}$ because $\operatorname{Pf}\left(H i \tau_{x}\right)=\operatorname{Pf}\left(H \tau_{x}\right)$ for matrices of size $4 n$ with $n$ an integer number. In the fully numerical approach, we set up the Hamiltonian for the superconductor subject to the Zeeman field and including the impurity potential, and use a supercell method to obtain $H\left(k_{x}\right)$ of an (infinite) impurity chain along the $x$ direction. For the calculation of the 
invariant using Eq. (7) the supercell Hamiltonian needs to be constructed for the two time-reversal invariant momenta, $k_{x}=$ $0, \pi / a_{0}$, corresponding to periodic or antiperiodic boundary conditions. Finally, the Pfaffian is calculated using an efficient numerical algorithm [42].

The effective Hamiltonian Eq. (5) inherits the symmetries from the bulk Hamiltonian in Eq. (2), i.e., it satisfies the particle-hole symmetry $\tau_{x} H_{\text {eff }}^{*}\left(-k_{x}\right) \tau_{x}=-H_{\text {eff }}\left(k_{x}\right)$, which can be read from Eq. (5) by using that also the other matrices in the expression obey the same symmetry, e.g., $\tau_{x} \hat{U}^{*} \tau_{x}=$ $-\hat{U}, \tau_{x} \tilde{G}^{*} \tau_{x}=\tilde{G}, \tau_{x} G_{I}^{*}\left(-k_{x}\right) \tau_{x}=-G_{I}\left(k_{x}\right)$, which can be derived from the original property $\tau_{x}\left[H_{\mathrm{BdG}}(\mathbf{p})+H_{\mathrm{Z}}\right]^{*} \tau_{x}=$ $-\left[H_{\mathrm{BdG}}(\mathbf{p})+H_{\mathrm{Z}}\right]$ of the bulk Hamiltonian. Therefore, the effective Hamiltonian can be used to calculate the topological invariant using Eq. (7), while the numerical effort is greatly reduced because of the small size of the corresponding matrices.

\section{APPENDIX D: TOPOLOGICAL GAP}

For the calculation of the topological gap, i.e., the minimal positive eigenvalue of the supercell Hamiltonian as a function of $k_{x}$, we calculate the eigenvalues for a grid of a few $k_{x}$ points between 0 and $\pi / a_{0}$, select the $k_{x}$ with the smallest positive eigenvalue, and then use an iterative procedure to find the smallest positive eigenvalue by a bisection bracketing algorithm to obtain $\Delta_{\text {topo }}$. This procedure is is implemented for both the supercell and the effective Hamiltonian approach to investigate the reliability of the approximation in deriving Eq. (5), see the Supplemental Material [40]. Finding very good agreement, we show in the main text only results stemming from the effective Hamiltonian since the calculation of eigenvalues is orders of magnitude faster once the expansion coefficients [Eqs. (B7) and (B8)] for $H_{\text {eff }}\left(k_{x}\right)$ have been calculated.
[1] C. Nayak, S. H. Simon, A. Stern, M. Freedman, and S. Das Sarma, Non-Abelian anyons and topological quantum computation, Rev. Mod. Phys. 80, 1083 (2008).

[2] S. D. Sarma, M. Freedman, and C. Nayak, Majorana zero modes and topological quantum computation, npj Quantum Inf. 1, 15001 (2015).

[3] A. Y. Kitaev, Unpaired Majorana fermions in quantum wires, Phys. Usp. 44, 131 (2001).

[4] R. M. Lutchyn, J. D. Sau, and S. Das Sarma, Majorana Fermions and a Topological Phase Transition in Semiconductor-Superconductor Heterostructures, Phys. Rev. Lett. 105, 077001 (2010).

[5] Y. Oreg, G. Refael, and F. von Oppen, Helical Liquids and Majorana Bound States in Quantum Wires, Phys. Rev. Lett. 105, 177002 (2010).

[6] V. Mourik, K. Zuo, S. M. Frolov, S. R. Plissard, E. P. A. M. Bakkers, and L. P. Kouwenhoven, Signatures of Majorana fermions in hybrid superconductor-semiconductor nanowire devices, Science 336, 1003 (2012).

[7] R. M. Lutchyn, E. P. A. M. Bakkers, L. P. Kouwenhoven, P. Krogstrup, C. M. Marcus, and Y. Oreg, Majorana zero modes in superconductor-semiconductor heterostructures, Nat. Rev. Mater. 3, 52 (2018).

[8] T.-P. Choy, J. M. Edge, A. R. Akhmerov, and C. W. J. Beenakker, Majorana fermions emerging from magnetic nanoparticles on a superconductor without spin-orbit coupling, Phys. Rev. B 84, 195442 (2011).

[9] I. Martin and A. F. Morpurgo, Majorana fermions in superconducting helical magnets, Phys. Rev. B 85, 144505 (2012).

[10] F. Pientka, L. I. Glazman, and F. von Oppen, Topological superconducting phase in helical Shiba chains, Phys. Rev. B 88, 155420 (2013).

[11] P. M. R. Brydon, S. Das Sarma, H.-Y. Hui, and J. D. Sau, Topological Yu-Shiba-Rusinov chain from spin-orbit coupling, Phys. Rev. B 91, 064505 (2015).

[12] L. Kimme and T. Hyart, Existence of zero-energy impurity states in different classes of topological insulators and superconductors and their relation to topological phase transitions, Phys. Rev. B 93, 035134 (2016).
[13] I. Sahlberg, A. Westström, K. Pöyhönen, and T. Ojanen, Engineering one-dimensional topological phases on $p$-wave superconductors, Phys. Rev. B 95, 184512 (2017).

[14] S. Nadj-Perge, I. K. Drozdov, B. A. Bernevig, and A. Yazdani, Proposal for realizing Majorana fermions in chains of magnetic atoms on a superconductor, Phys. Rev. B 88, 020407(R) (2013).

[15] S. Nadj-Perge, I. K. Drozdov, J. Li, H. Chen, S. Jeon, J. Seo, A. H. MacDonald, B. A. Bernevig, and A. Yazdani, Observation of Majorana fermions in ferromagnetic atomic chains on a superconductor, Science 346, 602 (2014).

[16] M. Ruby, F. Pientka, Y. Peng, F. von Oppen, B. W. Heinrich, and K. J. Franke, End States and Subgap Structure in ProximityCoupled Chains of Magnetic Adatoms, Phys. Rev. Lett. 115, 197204 (2015).

[17] R. Pawlak, M. Kisiel, J. Klinovaja, T. Meier, S. Kawai, T. Glatzel, D. Loss, and E. Meyer, Probing atomic structure and Majorana wavefunctions in mono-atomic Fe chains on superconducting Pb surface, npj Quantum Inf. 2, 16035 (2016).

[18] H. Kim, A. Palacio-Morales, T. Posske, L. Rózsa, K. Palotás, L. Szunyogh, M. Thorwart, and R. Wiesendanger, Toward tailoring Majorana bound states in artificially constructed magnetic atom chains on elemental superconductors, Sci. Adv. 4, eaar5251 (2018).

[19] H. Zhang, D. E. Liu, M. Wimmer, and L. P. Kouwenhoven, Next steps of quantum transport in Majorana nanowire devices, Nat. Commun. 10, 5128 (2019).

[20] S. Nakosai, J. C. Budich, Y. Tanaka, B. Trauzettel, and N. Nagaosa, Majorana Bound States and Nonlocal Spin Correlations in a Quantum Wire on an Unconventional Superconductor, Phys. Rev. Lett. 110, 117002 (2013).

[21] V. Kaladzhyan, J. Röntynen, P. Simon, and T. Ojanen, Topological state engineering by potential impurities on chiral superconductors, Phys. Rev. B 94, 060505(R) (2016).

[22] T. Neupert, A. Yazdani, and B. A. Bernevig, Shiba chains of scalar impurities on unconventional superconductors, Phys. Rev. B 93, 094508 (2016).

[23] V. Kaladzhyan, C. Bena, and P. Simon, Topology from triviality, Phys. Rev. B 97, 104512 (2018). 
[24] N. Sedlmayr, V. Kaladzhyan, and C. Bena, New tools to determine the topological character of Shiba chains, arXiv:2102.02214.

[25] J. Alicea, Y. Oreg, G. Refael, F. von Oppen, and M. P. A. Fisher, Non-Abelian statistics and topological quantum information processing in 1D wire networks, Nat. Phys. 7, 412 (2011).

[26] T. Hyart, B. van Heck, I. C. Fulga, M. Burrello, A. R. Akhmerov, and C. W. J. Beenakker, Flux-controlled quantum computation with Majorana fermions, Phys. Rev. B 88, 035121 (2013).

[27] J. Li, T. Neupert, B. A. Bernevig, and A. Yazdani, Manipulating Majorana zero modes on atomic rings with an external magnetic field, Nat. Commun. 7, 10395 (2016).

[28] D. Aasen, M. Hell, R. V. Mishmash, A. Higginbotham, J. Danon, M. Leijnse, T. S. Jespersen, J. A. Folk, C. M. Marcus, K. Flensberg, and J. Alicea, Milestones Toward Majorana-Based Quantum Computing, Phys. Rev. X 6, 031016 (2016).

[29] A. P. Mackenzie, T. Scaffidi, C. W. Hicks, and Y. Maeno, Even odder after twenty-three years: The superconducting order parameter puzzle of $\mathrm{Sr}_{2} \mathrm{RuO}_{4}$, npj Quantum Mater. 2, 40 (2017).

[30] S. A. Kivelson, A. C. Yuan, B. Ramshaw, and R. Thomale, A proposal for reconciling diverse experiments on the superconducting state in $\mathrm{Sr}_{2} \mathrm{RuO}_{4}$, npj Quantum Mater. 5, 43 (2020).

[31] A. Pustogow, Y. Luo, A. Chronister, Y.-S. Su, D. A. Sokolov, F. Jerzembeck, A. P. Mackenzie, C. W. Hicks, N. Kikugawa, S. Raghu, E. D. Bauer, and S. E. Brown, Constraints on the superconducting order parameter in $\mathrm{Sr}_{2} \mathrm{RuO}_{4}$ from oxygen-17 nuclear magnetic resonance, Nature (London) 574, 72 (2019).

[32] A. J. Leggett and Y. Liu, Symmetry properties of superconducting order parameter in $\mathrm{Sr}_{2} \mathrm{RuO}_{4}$, J. Supercond. Novel Magn. 34, 1647 (2020).

[33] A. Chronister, A. Pustogow, N. Kikugawa, D. A. Sokolov, F. Jerzembeck, C. W. Hicks, A. P. Mackenzie, E. D. Bauer, and S. E. Brown, Evidence for even parity unconventional superconductivity in $\mathrm{Sr}_{2} \mathrm{RuO}_{4}$, Proc. Natl. Acad. Sci. USA 118, e2025313118 (2021).

[34] E. R. Schemm, W. J. Gannon, C. M. Wishne, W. P. Halperin, and A. Kapitulnik, Observation of broken time-reversal symmetry in the heavy-fermion superconductor $\mathrm{UPt}_{3}$, Science 345, 190 (2014).

[35] R. Joynt and L. Taillefer, The superconducting phases of $\mathrm{UPt}_{3}$, Rev. Mod. Phys. 74, 235 (2002).

[36] S. Ran, C. Eckberg, Q.-P. Ding, Y. Furukawa, T. Metz, S. R. Saha, I.-L. Liu, M. Zic, H. Kim, J. Paglione, and N. P. Butch, Nearly ferromagnetic spin-triplet superconductivity, Science 365, 684 (2019).

[37] L. Jiao, S. Howard, S. Ran, Z. Wang, J. O. Rodriguez, M. Sigrist, Z. Wang, N. P. Butch, and V. Madhavan, Chiral superconductivity in heavy-fermion metal $\mathrm{UTe}_{2}$, Nature (London) 579, 523 (2020).

[38] Z. F. Weng, J. L. Zhang, M. Smidman, T. Shang, J. Quintanilla, J. F. Annett, M. Nicklas, G. M. Pang, L. Jiao, W. B. Jiang, Y. Chen, F. Steglich, and H. Q. Yuan, Two-Gap Superconductivity in $\mathrm{LaNiGa}_{2}$ with Nonunitary Triplet Pairing and Even Parity Gap Symmetry, Phys. Rev. Lett. 117, 027001 (2016).
[39] L. Kimme, T. Hyart, and B. Rosenow, Symmetry-protected topological invariant and Majorana impurity states in timereversal-invariant superconductors, Phys. Rev. B 91, 220501(R) (2015).

[40] See Supplemental Material at http://link.aps.org/supplemental/ 10.1103/PhysRevResearch.3.033049 for technical details related to the tight-binding models, the derivation and properties of the effective Hamiltonian, a comparison of numerical results obtained for the lattice model with those from the effective Hamiltonian, and additional discussions of routes towards movement, fusion, and braiding of Majorana zero modes. The supplemental material includes Refs. [43-54].

[41] T. Scaffidi and S. H. Simon, Large Chern Number and Edge Currents in $\mathrm{Sr}_{2} \mathrm{RuO}_{4}$, Phys. Rev. Lett. 115, 087003 (2015).

[42] M. Wimmer, Algorithm 923: Efficient numerical computation of the Pfaffian for dense and banded skew-symmetric matrices, ACM Trans. Math. Softw. 38, 30 (2012).

[43] A. T. Rømer, A. Kreisel, M. A. Müller, P. J. Hirschfeld, I. M. Eremin, and B. M. Andersen, Theory of strain-induced magnetic order and splitting of $T_{c}$ and $T_{\mathrm{TRSB}}$ in $\mathrm{Sr}_{2} \mathrm{RuO}_{4}$, Phys. Rev. B 102, 054506 (2020).

[44] T. Hyart, A. R. Wright, and B. Rosenow, Zeeman-field-induced topological phase transitions in triplet superconductors, Phys. Rev. B 90, 064507 (2014).

[45] C. W. J. Beenakker, Search for non-Abelian Majorana braiding statistics in superconductors, SciPost Phys. Lect. Notes, 15 (2020), doi: 10.21468/SciPostPhysLectNotes.15.

[46] S.-B. Zhang, W. B. Rui, A. Calzona, S.-J. Choi, A. P. Schnyder, and B. Trauzettel, Topological and holonomic quantum computation based on second-order topological superconductors, Phys. Rev. Research 2, 043025 (2020).

[47] Y. Tserkovnyak, A. Brataas, G. E. W. Bauer, and B. I. Halperin, Nonlocal magnetization dynamics in ferromagnetic heterostructures, Rev. Mod. Phys. 77, 1375 (2005).

[48] S. S. P. Parkin, M. Hayashi, and L. Thomas, Magnetic domainwall racetrack memory, Science 320, 190 (2008).

[49] F. Matsukura, Y. Tokura, and H. Ohno, Control of magnetism by electric fields, Nat. Nanotechnol. 10, 209 (2015).

[50] A. Hoffmann and S. D. Bader, Opportunities at the frontiers of spintronics, Phys. Rev. Appl. 4, 047001 (2015).

[51] Y. Tokura, K. Yasuda, and A. Tsukazaki, Magnetic topological insulators, Nat. Rev. Phys. 1, 126 (2019).

[52] A. Hirohata, K. Yamada, Y. Nakatani, I.-L. Prejbeanu, B. Diény, P. Pirro, and B. Hillebrands, Review on spintronics: Principles and device applications, J. Magn. Magn. Mater. 509, 166711 (2020).

[53] P. Bonderson, M. Freedman, and C. Nayak, MeasurementOnly Topological Quantum Computation, Phys. Rev. Lett. 101, 010501 (2008).

[54] T. Karzig, C. Knapp, R. M. Lutchyn, P. Bonderson, M. B. Hastings, C. Nayak, J. Alicea, K. Flensberg, S. Plugge, Y. Oreg, C. M. Marcus, and M. H. Freedman, Scalable designs for quasiparticle-poisoning-protected topological quantum computation with Majorana zero modes, Phys. Rev. B 95, 235305 (2017). 